\title{
NEW SERIES INVOLVING THE ZETA FUNCTION
}

\section{WU YUN-FEI}

\author{
(Received 29 January 2001)
}

\begin{abstract}
We evaluate sums of certain classes of new series involving the Riemann zeta function by using the theory of the double gamma function and a property of the gamma function. Relevant connections with various known results are also pointed out.
\end{abstract}

2000 Mathematics Subject Classification. 11M99.

1. Introduction. Barnes [1] defined the double gamma function $\Gamma_{2}=1 / G$ satisfying each of the following properties:

(a) $G(z+1)=\Gamma(z) G(z)$, for any complex number $z$;

(b) $G(1)=1$;

(c) As $n \rightarrow \infty$,

$$
\begin{aligned}
\log G(z+n+2)= & \frac{n+1+z}{2} \log (2 \pi)+\left[\frac{n^{2}}{2}+n+\frac{5}{12}+\frac{z^{2}}{2}+(n+1) z\right] \log n \\
& -\frac{3 z^{2}}{4}-n(1+z)-\log A+\frac{1}{12}+O\left(\frac{1}{n}\right)
\end{aligned}
$$

where $\Gamma$ is the well-known gamma function

$$
\Gamma^{-1}(z)=z e^{\gamma z} \prod_{n=1}^{\infty}\left(1+\frac{z}{n}\right) e^{-z / n},
$$

and $A$ is called Glaisher's (or Kinkelin's) constant defined by

$$
\log A=\lim _{n \rightarrow \infty}\left\{\log \left(1^{1} \cdot 2^{2} \cdots n^{n}\right)-\left(\frac{n^{2}}{2}+\frac{n}{2}+\frac{1}{12}\right) \log n+\frac{n^{2}}{4}\right\}
$$

the numerical value of $A$ being $1.282427130 \ldots$

From this definition, Barnes deduced

$$
\left\{\Gamma_{2}(z+1)\right\}^{-1}=G(z+1)+(2 \pi)^{z / 2} e^{-(1 / 2)\left[(1+\gamma) z^{2}+z\right]} \prod_{n=1}^{\infty}\left(1+\frac{z}{n}\right)^{n} e^{-z+z^{2} / 2 n},
$$

where $\gamma$ denotes the Euler-Mascheroni constant given by

$$
\gamma=\lim _{n \rightarrow \infty}\left(1+\frac{1}{2}+\cdots+\frac{1}{n}-\log n\right) \cong 0.577215664 \ldots
$$


It is also known that [1]

$$
\Gamma\left(\frac{1}{2}\right)=\pi^{1 / 2}, \quad G\left(\frac{1}{2}\right)=2^{1 / 24} \cdot \pi^{-1 / 4} \cdot e^{1 / 8} \cdot A^{-3 / 2} .
$$

The gamma function has the property

$$
\Gamma(s) \Gamma\left(s+\frac{1}{m}\right) \cdots \Gamma\left(s+\frac{m-1}{m}\right) \Gamma(1-m s)=\frac{2^{(m-1) / 2} \pi^{(m+1) / 2} m^{1 / 2-m s}}{\sin (m \pi s)} .
$$

The Riemann zeta function $\zeta(s)$ is defined by

$$
\zeta(s)=\sum_{n=1}^{\infty} \frac{1}{n^{s}}, \quad \operatorname{Res}(s)>1 .
$$

Indeed it is analytic for all $s$ except for a simple pole at $s=1$ with residue 1 .

In [2, 3] Choi, Srivastava, and Quine used the theory of the double gamma function to evaluate some series associated with the zeta function. Now in the present paper, we use the property of the gamma function and (2.23) and (2.70) in [2] to evaluate new series

$$
\begin{gathered}
\sum_{k=1}^{\infty} \frac{\zeta(2 k+1)}{(2 k+1)(2 k+2)} \alpha^{2 k}, \quad \sum_{k=2}^{\infty} \frac{\zeta(k)}{k(k+1)(k+2)} 2^{-k}, \quad \sum_{k=2}^{\infty} \frac{(-1)^{k} \zeta(k)}{k(k+1)(k+2)} 2^{-k}, \\
\sum_{k=2}^{\infty} \frac{\zeta(k)}{k(k+1)(k+2)(k+3)}, \quad \sum_{k=2}^{\infty} \frac{(-1)^{k} \zeta(k)}{k(k+1)(k+2)(k+3)},
\end{gathered}
$$

where $\alpha$ can be taken as $1 / 3,2 / 3,1 / 4,3 / 4,1 / 6,5 / 6$. And relevant connections with various known results are also pointed out.

2. Series involving the zeta function. Let $s=x$ and making $0<x<1$ in (1.2), we have

$$
\begin{aligned}
\log \Gamma(x)+\log x+\gamma x & =\sum_{n=1}^{\infty}\left[\frac{x}{n}-\log \left(1+\frac{x}{n}\right)\right] \\
& =\sum_{n=1}^{\infty} \sum_{k=2}^{\infty} \frac{(-1)^{k} x^{k}}{k n^{k}}=\sum_{k=2}^{\infty} \frac{(-1)^{k} x^{k}}{k} \sum_{n=1}^{\infty} \frac{1}{n^{k}} \\
& =\sum_{k=2}^{\infty} \frac{(-1)^{k} x^{k} \zeta(k)}{k} .
\end{aligned}
$$

Replacing $x$ by $1-x, 1-2 x, 1-3 x, x+1 / 2, x+1 / 3$, and $x+2 / 3$, we get

$$
\begin{gathered}
\log \Gamma(1-x)+\log (1-x)+\gamma(1-x)=\sum_{k=2}^{\infty} \frac{(-1)^{k}(1-x)^{k} \zeta(k)}{k}, \quad 0<x<1, \\
\log \Gamma(1-2 x)+\log (1-2 x)+\gamma(1-2 x)=\sum_{k=2}^{\infty} \frac{(-1)^{k}(1-2 x)^{k} \zeta(k)}{k}, \quad 0<x<\frac{1}{2}, \\
\log \Gamma(1-3 x)+\log (1-3 x)+\gamma(1-3 x)=\sum_{k=2}^{\infty} \frac{(-1)^{k}(1-3 x)^{k} \zeta(k)}{k}, \quad 0<x<\frac{1}{3},
\end{gathered}
$$




$$
\begin{array}{ll}
\log \Gamma\left(x+\frac{1}{2}\right)+\log \left(x+\frac{1}{2}\right)+\gamma\left(x+\frac{1}{2}\right)=\sum_{k=2}^{\infty} \frac{(-1)^{k}(x+1 / 2)^{k} \zeta(k)}{k}, & 0<x<\frac{1}{2}, \\
\log \Gamma\left(x+\frac{1}{3}\right)+\log \left(x+\frac{1}{3}\right)+\gamma\left(x+\frac{1}{3}\right)=\sum_{k=2}^{\infty} \frac{(-1)^{k}(x+1 / 3)^{k} \zeta(k)}{k}, & 0<x<\frac{2}{3}, \\
\log \Gamma\left(x+\frac{2}{3}\right)+\log \left(x+\frac{2}{3}\right)+\gamma\left(x+\frac{2}{3}\right)=\sum_{k=2}^{\infty} \frac{(-1)^{k}(x+2 / 3)^{k} \zeta(k)}{k}, & 0<x<\frac{1}{3} .
\end{array}
$$

Let $s=x, 0<x<1$ and making $m=1, m=2, m=3$ in (1.7), we get

$$
\begin{gathered}
\Gamma(x) \Gamma(1-x)=\frac{\pi}{\sin \pi x}, \\
\Gamma(x) \Gamma\left(x+\frac{1}{2}\right) \Gamma(1-2 x)=\frac{2^{1-2 x} \pi^{3 / 2}}{\sin (2 \pi x)}, \\
\Gamma(x) \Gamma\left(x+\frac{1}{3}\right) \Gamma\left(x+\frac{2}{3}\right) \Gamma(1-3 x)=\frac{2 \pi^{2} 3^{1 / 2-3 x}}{\sin (3 \pi x)} .
\end{gathered}
$$

Combining (2.1), (2.2), and (2.8), we obtain

$$
\begin{aligned}
& \log 2 \pi-\log (2 \sin \pi x)+\log [x(1-x)]+\gamma \\
&=\sum_{k=2}^{\infty} \frac{(-1)^{k}\left[x^{k}+(1-x)^{k}\right] \zeta(k)}{k}, \quad 0<x<1 .
\end{aligned}
$$

Combining (2.1), (2.3), (2.5), and (2.9), we obtain

$$
\begin{gathered}
\frac{3}{2} \log 2 \pi+\left(\frac{1}{2}-2 x\right) \log 2-\log [2 \sin (2 \pi x)]+\log \left[x\left(x+\frac{1}{2}\right)(1-2 x)\right]+\frac{3}{2} \gamma \\
=\sum_{k=2}^{\infty} \frac{(-1)^{k}\left[x^{k}+(x+1 / 2)^{k}+(1-2 x)^{k}\right] \zeta(k)}{k}, \quad 0<x<\frac{1}{2} .
\end{gathered}
$$

Combining (2.1), (2.4), (2.6), (2.7), and (2.10), we obtain

$$
\begin{gathered}
2 \log 2 \pi+\left(\frac{1}{2}-3 x\right) \log 3-\log [2 \sin (3 \pi x)]+\log \left[x\left(x+\frac{1}{3}\right)\left(x+\frac{2}{3}\right)(1-3 x)\right]+2 \gamma \\
=\sum_{k=2}^{\infty} \frac{(-1)^{k}\left[x^{k}+(x+1 / 3)^{k}+(x+2 / 3)^{k}+(1-3 x)^{k}\right] \zeta(k)}{k}, \quad 0<x<\frac{1}{3}
\end{gathered}
$$

On the other hand, recalling Euler formula

$$
\frac{\sin \pi x}{\pi x}=\prod_{n=1}^{\infty}\left(1-\frac{x^{2}}{n^{2}}\right), \quad 0<x<1,
$$


similarly, we get

$$
\begin{aligned}
\log (2 \sin \pi x)-\log (2 \pi x) & =-\sum_{k=1}^{\infty} \frac{x^{2 k} \zeta(2 k)}{k}, \\
\log [2 \sin \pi(1-x)]-\log [2 \pi(1-x)] & =-\sum_{k=1}^{\infty} \frac{(1-x)^{2 k} \zeta(2 k)}{k}, \\
\log [2 \sin \pi(1-2 x)]-\log [2 \pi(1-2 x)] & =-\sum_{k=1}^{\infty} \frac{(1-2 x)^{2 k} \zeta(2 k)}{k}, \\
\log [2 \sin \pi(1-3 x)]-\log [2 \pi(1-3 x)] & =-\sum_{k=1}^{\infty} \frac{(1-3 x)^{2 k} \zeta(2 k)}{k}, \\
\log \left[2 \sin \pi\left(x+\frac{1}{2}\right)\right]-\log \left[2 \pi\left(x+\frac{1}{2}\right)\right] & =-\sum_{k=1}^{\infty} \frac{(x+1 / 2)^{2 k} \zeta(2 k)}{k}, \\
\log \left[2 \sin \pi\left(x+\frac{1}{3}\right)\right]-\log \left[2 \pi\left(x+\frac{1}{3}\right)\right] & =-\sum_{k=1}^{\infty} \frac{(x+1 / 3)^{2 k} \zeta(2 k)}{k}, \\
\log \left[2 \sin \pi\left(x+\frac{2}{3}\right)\right]-\log \left[2 \pi\left(x+\frac{2}{3}\right)\right] & =-\sum_{k=1}^{\infty} \frac{(x+2 / 3)^{2 k} \zeta(2 k)}{k} .
\end{aligned}
$$

Combining (2.15) and (2.16), we obtain

$$
\log (2 \sin \pi x)-\log 2 \pi-\frac{1}{2} \log [x(1-x)]=-\sum_{k=1}^{\infty} \frac{\left[x^{2 k}+(1-x)^{2 k}\right] \zeta(2 k)}{2 k} .
$$

Noting that

$$
\log (2 \sin \pi x)+\log \left[2 \sin \pi\left(x+\frac{1}{2}\right)\right]=\log [2 \sin (2 \pi x)],
$$

and by combining (2.15), (2.17), and (2.19), we obtain

$$
\begin{aligned}
\log [2 \sin (2 \pi x)] & -\frac{3}{2} \log 2 \pi-\frac{1}{2} \log \left[x\left(x+\frac{1}{2}\right)(1-2 x)\right] \\
& =-\sum_{k=1}^{\infty} \frac{\left[x^{2 k}+(x+1 / 2)^{2 k}+(1-2 x)^{2 k}\right] \zeta(2 k)}{2 k} .
\end{aligned}
$$

Noting that

$$
\log (2 \sin \pi x)+\log \left[2 \sin \pi\left(x+\frac{1}{3}\right)\right]+\log \left[2 \sin \pi\left(x+\frac{2}{3}\right)\right]=\log [2 \sin (3 \pi x)],
$$

and by combining (2.15), (2.18), (2.20), and (2.21), we obtain

$$
\begin{aligned}
\log [2 \sin (3 \pi x)]-2 \log 2 \pi-\frac{1}{2} \log \left[x\left(x+\frac{1}{3}\right)\left(x+\frac{2}{3}\right)(1-3 x)\right] \\
=-\sum_{k=1}^{\infty} \frac{\left[x^{2 k}+(x+1 / 3)^{2 k}+(x+2 / 3)^{2 k}+(1-3 x)^{2 k}\right] \zeta(2 k)}{2 k} .
\end{aligned}
$$


Now combining (2.11) and (2.22), (2.12) and (2.24), (2.13) and (2.26), respectively, we get

$$
\begin{aligned}
& \frac{1}{2} \log [x(1-x)]+\gamma=-\sum_{k=1}^{\infty} \frac{\left[x^{2 k+1}+(1-x)^{2 k+1}\right] \zeta(2 k+1)}{2 k+1}, 0<x<1, \\
& \left(\frac{1}{2}-2 x\right) \log 2+\frac{1}{2} \log \left[x\left(x+\frac{1}{2}\right)(1-2 x)\right]+\frac{3}{2} \gamma \\
& =-\sum_{k=1}^{\infty} \frac{\left[x^{2 k+1}+(x+1 / 2)^{2 k+1}+(1-2 x)^{2 k+1}\right] \zeta(2 k+1)}{2 k+1}, \quad 0<x<\frac{1}{2}, \\
& \left(\frac{1}{2}-3 x\right) \log 3+\frac{1}{2} \log \left[x\left(x+\frac{1}{3}\right)\left(x+\frac{2}{3}\right)(1-3 x)\right]+2 \gamma \\
& =-\sum_{k=1}^{\infty} \frac{\left[x^{2 k+1}+(x+1 / 3)^{2 k+1}+(x+2 / 3)^{2 k+1}+(1-3 x)^{2 k+1}\right] \zeta(2 k+1)}{2 k+1}, 0<x<\frac{1}{3} .
\end{aligned}
$$

Integrating both sides of (2.27) from $x=0$ to $x=a$, we get

$$
\frac{1}{2} a \log a-\frac{1}{2}(1-a) \log (1-a)+a(\gamma-1)=-\sum_{k=1}^{\infty} \frac{\left[a^{2 k+2}-(1-a)^{2 k+2}+1\right] \zeta(2 k+1)}{(2 k+1)(2 k+2)}
$$

Making $a=1 / 2, a=1 / 3, a=1 / 4$, and $a=1 / 6$, we have

$$
\begin{gathered}
\sum_{k=1}^{\infty} \frac{\zeta(2 k+1)}{(2 k+1)(2 k+2)}=\frac{1}{2}(1-\gamma), \\
\sum_{k=1}^{\infty} \frac{\left[(2 / 3)^{2 k+2}-(1 / 3)^{2 k+2}\right] \zeta(2 k+1)}{(2 k+1)(2 k+2)}=-\frac{1}{3} \log 2+\frac{1}{6} \log 3+\frac{1}{6}(1-\gamma), \\
\sum_{k=1}^{\infty} \frac{\left[(3 / 4)^{2 k+2}-(1 / 4)^{2 k+2}\right] \zeta(2 k+1)}{(2 k+1)(2 k+2)}=\frac{1}{2} \log 2-\frac{3}{8} \log 3+\frac{1}{4}(1-\gamma), \\
\sum_{k=1}^{\infty} \frac{\left[(5 / 6)^{2 k+2}-(1 / 6)^{2 k+2}\right] \zeta(2 k+1)}{(2 k+1)(2 k+2)}=\frac{1}{3} \log 6-\frac{5}{12} \log 5+\frac{1}{3}(1-\gamma) .
\end{gathered}
$$

Integrating both sides of (2.28) from $x=0$ to $x=1 / 4$, we have

$$
\begin{aligned}
-\frac{9}{16} & \log 2+\frac{3}{8} \log 3-\frac{3}{8}(1-\gamma) \\
& =-\sum_{k=1}^{\infty} \frac{\left[(1 / 4)^{2 k+2}+(3 / 4)^{2 k+2}-3(1 / 2)^{2 k+3}+1 / 2\right] \zeta(2 k+1)}{(2 k+1)(2 k+2)} .
\end{aligned}
$$

Recalling the formula [2, page 113]

$$
\sum_{k=1}^{\infty} \frac{\zeta(2 k+1)}{(2 k+1)(2 k+2)} 2^{-2 k}=1+\frac{7}{6} \log 2-\frac{1}{2} \gamma-6 \log A,
$$


and combining (2.31), (2.35), and (2.36), we get

$$
\sum_{k=1}^{\infty} \frac{\left[(1 / 4)^{2 k+2}+(3 / 4)^{2 k+2}\right] \zeta(2 k+1)}{(2 k+1)(2 k+2)}=\frac{1}{2}+\log 2-\frac{3}{8} \log 3-\frac{5}{16} \gamma-\frac{9}{4} \log A .
$$

Solving (2.33) and (2.37), we obtain the formulas

$$
\begin{aligned}
& \sum_{k=1}^{\infty} \frac{\zeta(2 k+1)}{(2 k+1)(2 k+2)} 4^{-2 k}=2+4 \log 2-\frac{\gamma}{2}-18 \log A, \\
& \sum_{k=1}^{\infty} \frac{\zeta(2 k+1)}{(2 k+1)(2 k+2)}\left(\frac{3}{4}\right)^{2 k}=\frac{2}{3}+\frac{4}{3} \log 2-\frac{2}{3} \log 3-\frac{\gamma}{2}-2 \log A .
\end{aligned}
$$

Multiplying $1 / 3-x$ on both sides of (2.29), and integrating from $x=0$ to $x=1 / 3$, we have

$$
\begin{aligned}
-\frac{2}{9}-\frac{1}{9} \log 2+\frac{19}{108} \log 3+\frac{\gamma}{9}= & -\frac{8}{9} \sum_{k=1}^{\infty} \frac{\zeta(2 k+1)}{(2 k+1)(2 k+2)(2 k+3)} \\
& +\frac{1}{3} \sum_{k=1}^{\infty} \frac{\left[(1 / 3)^{2 k+2}+(2 / 3)^{2 k+2}-1 / 3\right] \zeta(2 k+1)}{(2 k+1)(2 k+2)} .
\end{aligned}
$$

Recalling the formula [2, page 118]

$$
\sum_{k=1}^{\infty} \frac{\zeta(2 k+1)}{(2 k+1)(2 k+2)(2 k+3)}=\frac{3}{8}-\frac{\gamma}{6}-\log A,
$$

and combining (2.31), (2.39), and (2.40), we have

$$
\sum_{k=1}^{\infty} \frac{\left[(1 / 3)^{2 k+2}+(2 / 3)^{2 k+2}\right] \zeta(2 k+1)}{(2 k+1)(2 k+2)}=\frac{1}{2}-\frac{1}{3} \log 2+\frac{19}{36} \log 3-\frac{5}{18} \gamma-\frac{8}{3} \log A
$$

Solving (2.32) and (2.41), we obtain the formulas

$$
\begin{aligned}
& \sum_{k=1}^{\infty} \frac{\zeta(2 k+1)}{(2 k+1)(2 k+2)} 3^{-2 k}=\frac{3}{2}+\frac{13}{8} \log 3-\frac{\gamma}{2}-12 \log A, \\
& \sum_{k=1}^{\infty} \frac{\zeta(2 k+1)}{(2 k+1)(2 k+2)}\left(\frac{2}{3}\right)^{2 k}=\frac{3}{4}-\frac{3}{4} \log 2+\frac{25}{32} \log 3-\frac{\gamma}{2}-3 \log A .
\end{aligned}
$$

Integrating both sides of (2.29) from $x=0$ to $x=1 / 6$, we get

$$
\begin{aligned}
& -\frac{1}{3}-\log 2+\frac{1}{24} \log 3+\frac{5}{12} \log 5+\frac{\gamma}{3} \\
& =-\sum_{k=1}^{\infty} \frac{\left[(1 / 6)^{2 k+2}+(5 / 6)^{2 k+2}-(1 / 3)^{2 k+2}-(2 / 3)^{2 k+2}+(2 / 3)(1 / 2)^{2 k+2}+1 / 3\right] \zeta(2 k+1)}{(2 k+1)(2 k+2)} .
\end{aligned}
$$


Combining (2.31), (2.36), (2.41), and (2.43), we have

$$
\begin{aligned}
\sum_{k=1}^{\infty} & \frac{\left[(1 / 6)^{2 k+2}+(5 / 6)^{2 k+2}\right] \zeta(2 k+1)}{(2 k+1)(2 k+2)} \\
& =\frac{1}{2}+\frac{17}{36} \log 2+\frac{35}{72} \log 3-\frac{5}{12} \log 5-\frac{13}{36} \gamma-\frac{5}{3} \log A
\end{aligned}
$$

Solving (2.44) and (2.34), we obtain the formulas

$$
\begin{aligned}
\sum_{k=1}^{\infty} \frac{\zeta(2 k+1)}{(2 k+1)(2 k+2)} 6^{-2 k} & =3+\frac{5}{2} \log 2+\frac{11}{4} \log 3-\frac{\gamma}{2}-30 \log A, \\
\sum_{k=1}^{\infty} \frac{\zeta(2 k+1)}{(2 k+1)(2 k+2)}\left(\frac{5}{6}\right)^{2 k} & =\frac{3}{5}+\frac{29}{50} \log 2+\frac{59}{100} \log 3-\frac{3}{5} \log 5-\frac{\gamma}{2}-\frac{6}{5} \log A .
\end{aligned}
$$

Multiplying $1 / 2-x$ on both sides of (2.27), and integrating from $x=0$ to $x=1 / 2$, we have

$$
-\frac{1}{16}-\frac{1}{8} \log 2+\frac{\gamma}{8}=-\frac{1}{2} \sum_{k=1}^{\infty} \frac{\zeta(2 k+1)}{(2 k+1)(2 k+2)}-\sum_{k=1}^{\infty} \frac{\left[(1 / 2)^{2 k+2}-1\right] \zeta(2 k+1)}{(2 k+1)(2 k+2)(2 k+3)}
$$

Combining (2.31), (2.40), and (2.46), we obtain the formula

$$
\sum_{k=1}^{\infty} \frac{\zeta(2 k+1)}{(2 k+1)(2 k+2)(2 k+3)} 2^{-2 k}=\frac{3}{4}+\frac{1}{2} \log 2-\frac{\gamma}{6}-4 \log A .
$$

Multiplying $(1 / 2-x)^{2}$ on both sides of (2.27), and integrating from $x=0$ to $x=1 / 2$, we have

$$
\begin{aligned}
-\frac{1}{18}+\frac{\gamma}{24}= & -\frac{1}{4} \sum_{k=1}^{\infty} \frac{\zeta(2 k+1)}{(2 k+1)(2 k+2)}+\sum_{k=1}^{\infty} \frac{\zeta(2 k+1)}{(2 k+1)(2 k+2)(2 k+3)} \\
& -2 \sum_{k=1}^{\infty} \frac{\zeta(2 k+1)}{(2 k+1)(2 k+2)(2 k+3)(2 k+4)} .
\end{aligned}
$$

Combining (2.31), (2.40), and (2.48), we obtain the formula

$$
\sum_{k=1}^{\infty} \frac{\zeta(2 k+1)}{(2 k+1)(2 k+2)(2 k+3)(2 k+4)}=\frac{11}{72}-\frac{\gamma}{24}-\frac{1}{2} \log A .
$$

On the other hand, noting that Fourier series

$$
\log (2 \sin \pi x)=-\sum_{k=1}^{\infty} \frac{\cos (2 k \pi x)}{k}, \quad 0<x<1,
$$


and integrating by parts, we have

$$
\begin{aligned}
\int_{0}^{1}(1-x)^{2} \log (2 \sin \pi x) d x & =-\int_{0}^{1}(1-x)^{2} \sum_{k=1}^{\infty} \frac{\cos (2 k \pi x)}{k} d x \\
& =-\frac{1}{2 \pi} \int_{0}^{1}(1-x)^{2} d\left(\sum_{k=1}^{\infty} \frac{\sin (2 k \pi x)}{k^{2}}\right) \\
& =\frac{1}{2 \pi^{2}} \int_{0}^{1}(1-x) d\left(\sum_{k=1}^{\infty} \frac{\cos (2 k \pi x)}{k^{3}}\right)=-\frac{1}{2 \pi^{2}} \zeta(3) .
\end{aligned}
$$

Similarly, we have

$$
\begin{gathered}
\int_{0}^{1} \log (2 \sin \pi x) d x=\int_{0}^{1}(1-x) \log (2 \sin \pi x) d x=\int_{0}^{1 / 2} \log (2 \sin \pi x) d x=0, \\
\int_{0}^{1 / 2}\left(\frac{1}{2}-x\right) \log (2 \sin \pi x) d x=-\frac{7}{16 \pi^{2}} \zeta(3) .
\end{gathered}
$$

Integrating both sides of (2.15) from $x=0$ to $x=1$, and using (2.52), we get

$$
\sum_{k=1}^{\infty} \frac{\zeta(2 k)}{2 k(2 k+1)}=\frac{1}{2}(\log 2 \pi-1) .
$$

Combining (2.31) and (2.54), we obtain the formulas

$$
\begin{gathered}
\sum_{k=2}^{\infty} \frac{\zeta(k)}{k(k+1)}=\frac{1}{2}(\log 2 \pi-\gamma), \\
\sum_{k=2}^{\infty} \frac{(-1)^{k} \zeta(k)}{k(k+1)}=-1+\frac{1}{2} \log 2 \pi+\frac{\gamma}{2} .
\end{gathered}
$$

Integrating both sides of (2.15) from $x=0$ to $x=1 / 2$, and using (2.52), we get

$$
\sum_{k=1}^{\infty} \frac{\zeta(2 k)}{2 k(2 k+1)} 2^{-2 k}=\frac{1}{2}(\log \pi-1) .
$$

Combining (2.36) and (2.56), we obtain the formulas

$$
\begin{gathered}
\sum_{k=2}^{\infty} \frac{\zeta(k)}{k(k+1)} 2^{-k}=\frac{1}{2} \log \pi+\frac{7}{12} \log 2-\frac{\gamma}{4}-3 \log A, \\
\sum_{k=2}^{\infty} \frac{(-1)^{k} \zeta(k)}{k(k+1)} 2^{-k}=-1+\frac{1}{2} \log \pi-\frac{7}{12} \log 2+\frac{\gamma}{4}+3 \log A .
\end{gathered}
$$

Multiplying $1-x$ on both sides of (2.15), and integrating from $x=0$ to $x=1$, and using (2.52), we get

$$
\sum_{k=1}^{\infty} \frac{\zeta(2 k)}{2 k(2 k+1)(2 k+2)}=-\frac{3}{8}+\frac{1}{4} \log 2 \pi .
$$


Combining (2.58) and (2.40), we obtain the formulas

$$
\begin{gathered}
\sum_{k=2}^{\infty} \frac{\zeta(k)}{k(k+1)(k+2)}=\frac{1}{4} \log 2 \pi-\frac{\gamma}{6}-\log A, \\
\sum_{k=2}^{\infty} \frac{(-1)^{k} \zeta(k)}{k(k+1)(k+2)}=-\frac{3}{4}+\frac{1}{4} \log 2 \pi+\frac{\gamma}{6}+\log A .
\end{gathered}
$$

Multiplying $1 / 2-x$ on both sides of (2.15), and integrating from $x=0$ to $x=1 / 2$, and using (2.53), we get

$$
\sum_{k=1}^{\infty} \frac{\zeta(2 k)}{2 k(2 k+1)(2 k+2)} 2^{-2 k}=-\frac{3}{8}+\frac{1}{4} \log \pi+\frac{7}{8 \pi^{2}} \zeta(3) .
$$

Combining (2.60) and (2.47), we obtain the formulas

$$
\begin{aligned}
& \sum_{k=2}^{\infty} \frac{\zeta(k)}{k(k+1)(k+2)} 2^{-k}=\frac{1}{4} \log 2 \pi-\frac{\gamma}{12}+\frac{7}{8 \pi^{2}} \zeta(3)-2 \log A, \\
& \sum_{k=2}^{\infty} \frac{(-1)^{k} \zeta(k)}{k(k+1)(k+2)} 2^{-k}=-\frac{3}{4}+\frac{1}{4} \log \frac{\pi}{2}+\frac{\gamma}{12}+\frac{7}{8 \pi^{2}} \zeta(3)+2 \log A .
\end{aligned}
$$

Multiplying $(1-x)^{2}$ on both sides of (2.15), and integrating from $x=0$ to $x=1$, and using (2.51), we get

$$
\sum_{k=1}^{\infty} \frac{\zeta(2 k)}{2 k(2 k+1)(2 k+2)(2 k+3)}=-\frac{11}{72}+\frac{1}{12} \log 2 \pi+\frac{1}{8 \pi^{2}} \zeta(3) .
$$

Combining (2.62) and (2.49), we obtain the formulas

$$
\begin{aligned}
& \sum_{k=2}^{\infty} \frac{\zeta(k)}{k(k+1)(k+2)(k+3)}=\frac{1}{12} \log 2 \pi-\frac{\gamma}{24}+\frac{1}{8 \pi^{2}} \zeta(3)-\frac{1}{2} \log A, \\
& \sum_{k=2}^{\infty} \frac{(-1)^{k} \zeta(k)}{k(k+1)(k+2)(k+3)}=-\frac{11}{36}+\frac{1}{12} \log 2 \pi+\frac{\gamma}{24}+\frac{1}{8 \pi^{2}} \zeta(3)+\frac{1}{2} \log A .
\end{aligned}
$$

\section{REFERENCES}

[1] E. W. Barnes, The theory of the G-function, Quart. J. Pure Appl. Math. 31 (1899), 264-314.

[2] J. Choi and H. M. Srivastava, Sums associated with the zeta function, J. Math. Anal. Appl. 206 (1997), no. 1, 103-120. MR 97i:11092. Zbl 0869.11067.

[3] J. Choi, H. M. Srivastava, and J. R. Quine, Some series involving the zeta function, Bull. Austral. Math. Soc. 51 (1995), no. 3, 383-393. MR 96d:11090. Zbl 0830.11030.

Wu Yun-Fei: Department of Mathematics, Ningbo University, Ningbo, Zhejiang 315211, CHINA

E-mail address: yunfeiwu@163.net 


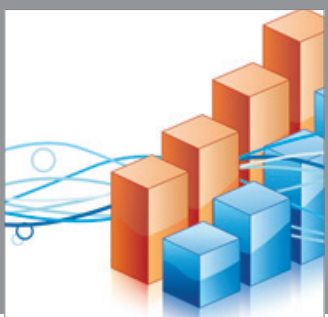

Advances in

Operations Research

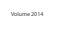

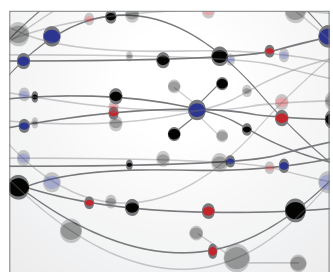

\section{The Scientific} World Journal
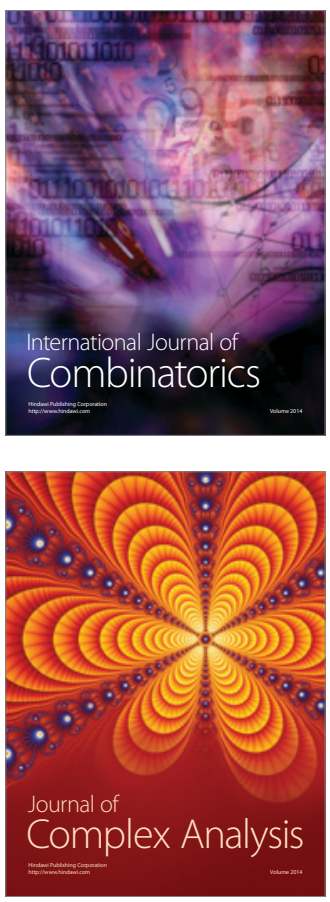

International Journal of

Mathematics and

Mathematical

Sciences
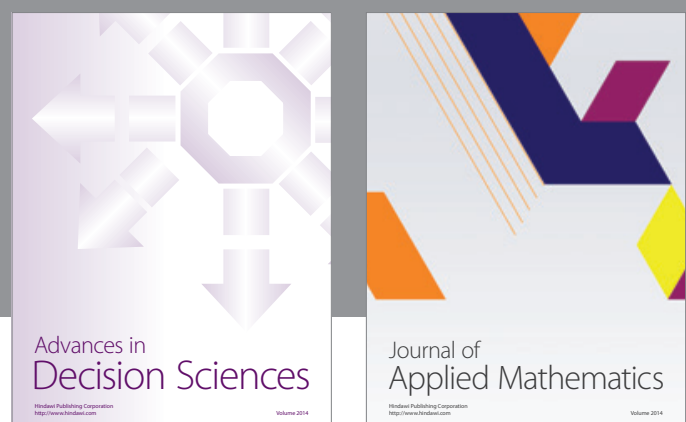

Journal of

Applied Mathematics
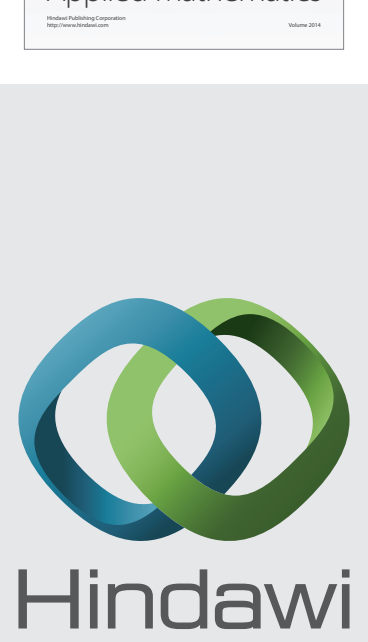

Submit your manuscripts at http://www.hindawi.com
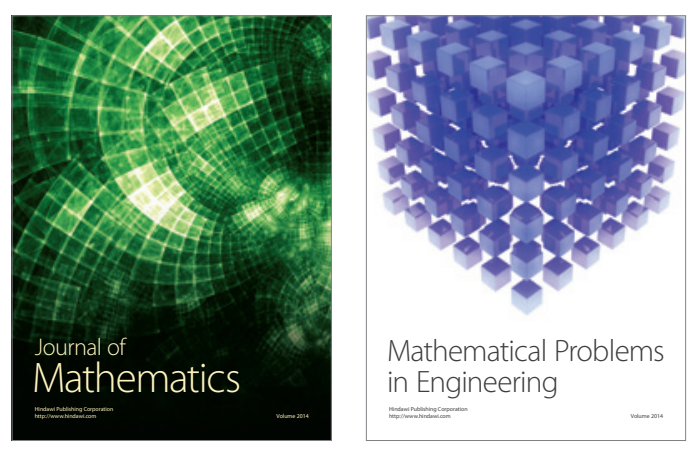

Mathematical Problems in Engineering
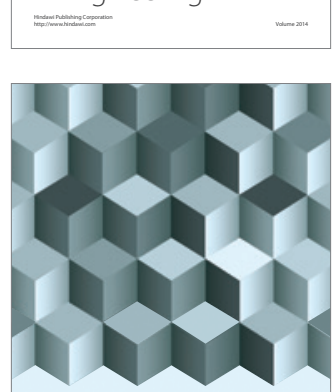

Journal of

Function Spaces
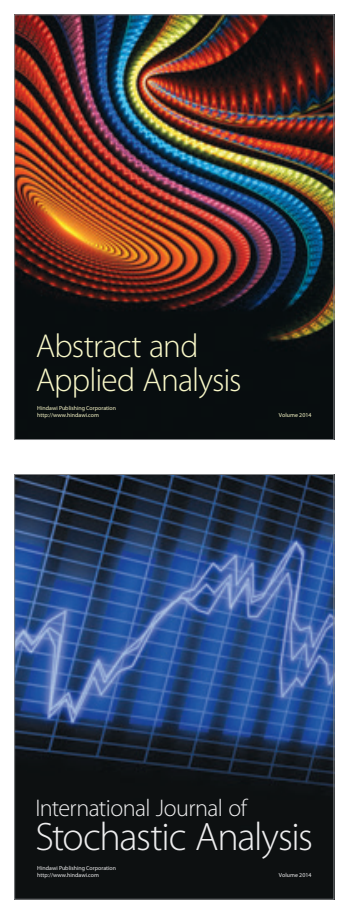

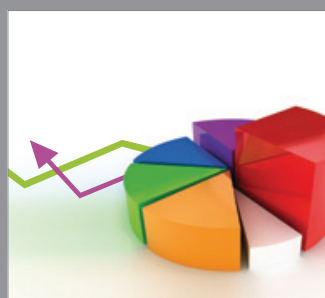

ournal of

Probability and Statistics

Promensencen
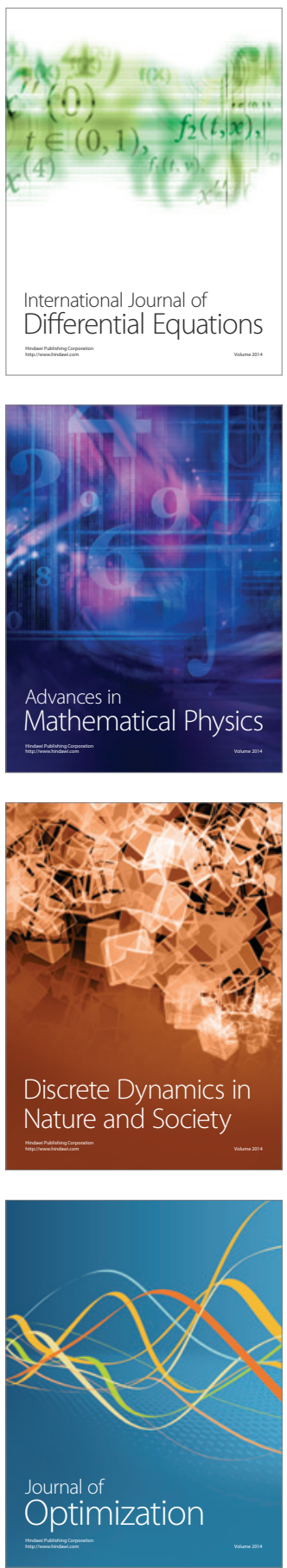